Pacific Journal of Mathematics

COMPOSITION PROPERTIES OF PROJECTIVE HOMOTOPY

SAMUEL S. Fed 


\title{
COMPOSITION PROPERTIES OF PROJECTIVE HOMOTOPY CLASSES
}

\author{
S. Feder, S. Gitler and K. Y. Lam
}

1. Introduction. A homotopy class $x \in \pi_{q}(X)$ is said to be projective on $X$, or projectively carried by $X$, if it can be represented by a map that factors through the projective space $p^{q}$, as shown in diagram (I), where $\hat{\pi}$ is the double covering map.

(I)

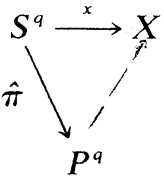

(II)

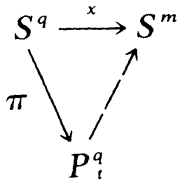

$$
(t \leqq m)
$$

When $x$ is a stable homotopy class of spheres, it is of interest to ask for the values of $m$ such that $x$ be projective on $S^{m}$. Since $S^{m}$ is $(t-1)$-connected for $t \leqq m$, this amounts to the factorisation problem posed in diagram (II) above, where $\pi$ is $\hat{\pi}$ followed by the collapsing map from $P^{q}$ to the truncated projective space $P_{t}^{q}=P^{q} / P^{t-1}$. We give an answer to this problem when $x$ is a generator of the image of the $J$-homomorphism.

In recent years the problem of projective classes has been studied in [3], [8], [10], [13], and [14]. We refer especially to [8], in which the authors determine, for classes up to the 28 -stem, the various spheres that can carry them projectively. We also refer to [10], where the authors prove among other things, that every stable homotopy class of spheres is carried projectively by some sphere.

Let $u, v$ be classes in stable stems of spheres, and suppose $x$ is projectively carried by $X$. For specific $u$ and $v$, it sometimes turns out that the composition $x u$, or a representative $x^{\prime}$ of the secondary composition $\langle x, u, v\rangle$ (i.e., Toda bracket), will also be carried by $X$ projectively. In this paper we prove the following typical results.

THEOREM 5.1. Let $x \in \pi_{q}(X)$ be projective on the $(m-1)$-connected space $X$, where $q \leqq 2 m-4$. Then $x \eta$ is projective on $X$.

THEOREM 6.2. Let $x \in \pi_{q}(X)$ be projective on the $(m-1)$ connected space $X$, where $q \leqq 2 m-10$ and $q \equiv 3(\bmod 4)$. If the Toda bracket $\langle x, 8 \sigma, 2\rangle$ is defined, then all its representatives are projective on $X$.

As an application we deduce Mahowald's Theorem D [7, p. 4] on the vanishing of certain Whitehead products, namely 
THEOREM (Mahowald). Let $\beta$ generate the image of the $J$ homomorphism in the $s$-stem. Then $\left[\iota_{n}, \beta\right]=0$ on $S^{n}$, provided $n$ and $s$ satisfy $3 \leqq \nu_{2}(n+s+2) \leqq \phi(s)$.

Here the numerical functions are defined as follows. $\nu_{2}(m)$ is the exponent of 2 in the factorization of $m$. For any integer number $s, \phi(s)$ denotes the number of integers in the closed interval $[1, s]$ which are congruent to $0,1,2$ or 4 modulo 8 . By [1], $2^{\phi(s)}$ is precisely the order of $\widetilde{K O}\left(P^{s}\right)$ or $J\left(P^{s}\right)$.

As a matter of fact, this paper is an outgrowth of our attempt to understand [7], in particular Theorem D. We thank Mark Mahowald warmly for many conversations and correspondences concerning his memoir.

The technical tools used to prove Theorems (5.1) and (6.2) are the "compression theorems" in $\$ 4$. These theorems deal with certain self-maps of truncated projective spaces. They help us pin down some facts about composition of the $\operatorname{Im} J$ generators, and facilitate the handling of Toda brackets. Perhaps these theorems have some independent interest, and could be read on their own right.

2. Preliminaries, notations and examples. Odd torsion elements being unimportant for our purpose, $\pi_{*}(X)$ in this paper will always mean the 2-primary component of the homotopy groups of $X$. For example, the 1st., 3rd., and 7th. stable homotopy groups of spheres are, for us, cyclic of order 2,8 , and 16 respectively, with generators $\eta, \nu$ and $\sigma$. A generator $\beta$ of $\operatorname{Im} J$ has order equal to a power of 2, as given by the Adams' conjecture ([6], [12]).

We fix some notation for maps between various spaces. The letter $c$ always denotes a map collapsing a subspace of some truncated projective space to a point. The double covering map $S^{q} \rightarrow P^{q}$ is $\hat{\pi}$, and $\pi$ will always denote a map which is $\hat{\pi}$ followed by a collapsing map c. Finally, inclusions between truncated projective spaces are usually indicated by $i$, or by an unmarked arrow.

We shall need the preliminary results $2.1-2.6$ below, whose details can be found in [8] or [14].

PROPOSITION 2.1. The class $2 \iota_{m}$ is projective on $S^{m}$ if and only if $m$ is odd.

Proposition 2.2. The classes $\eta, \nu$, and $\sigma$ are projective on $S^{m}$ if and only if

$$
\begin{aligned}
& m \equiv 2(\bmod 4) \text { in the case of } \eta \\
& m \equiv 4(\bmod 8) \text { in the case of } \nu ; \text { and } \\
& m \equiv 8(\bmod 16) \text { in the case of } \sigma ; \text { respectively. }
\end{aligned}
$$


For example, if $m \not \equiv 4(\bmod 8)$, then $\nu: S^{m+3} \rightarrow S^{m}$ cannot be factored as $S^{m+3} \stackrel{\hat{m}}{\rightarrow} P^{m+3} \stackrel{g}{\rightarrow} S^{m}$, otherwise the induced cofibre map $g^{\prime}: P^{m+4} \rightarrow S^{m} \cup_{\nu} e^{m+4}$ will contradict the action of the Steenrod squares.

Proposition 2.3. For $m \geqq k+2$, the projective homotopy classes in $\pi_{k+m}\left(S^{m}\right)$ form a subgroup $\pi_{k+m}^{\mathrm{proj}}\left(S^{m}\right)$.

For the next proposition let $M_{k+1}$ denote any suitable multiple of $2^{\phi(k+1)}$, and write $n=M_{k+1}-k-m-2$. Let $i: S^{n} \rightarrow P_{n}$ be the inclusion of $S^{n}$ into the bottom cell of $P_{n}=P^{\infty} / P^{n-1}$. With the same hypothesis as in (2.3) one has

Proposition 2.4. The stable class $x$ belongs to $\pi_{k+m}^{\mathrm{proj}}\left(S^{m}\right)$ if and only if the following composition is homotopically trivial:

$$
S^{k+n} \stackrel{x}{\rightarrow} S^{n} \stackrel{!}{\rightarrow} P_{n}
$$

This proposition is proved in [8], in a straight-forward manner, essentially by taking the $S$-dual of diagram (II), with $t=m$. From now on, we shall refer to the triviality of $i_{*}(x)$ by simply saying that $x$ "vanishes in $P_{n}$ ".

Proposition 2.5. The suspension homomorphism induces an isomorphism $\pi_{k+m}^{\text {proj }}\left(S^{m}\right) \cong \pi_{k+m+M}^{\text {proj }}\left(S^{m+M}\right)$ for any multiple $M$ of $2^{\phi(k+1)}$.

As a special example of projective homotopy classes consider the stable 8-stem $\pi_{8}^{s}=Z_{2} \oplus Z_{2}$, with generators $\bar{\nu}$ and $\sigma \eta$. The two elements $\bar{\nu}$ and $\bar{\nu}+\eta \sigma$ make up the full Toda bracket $\left\langle\nu^{2}, 2, \eta\right\rangle$. (See [15].) Moreover we have (c.f. [8]):

Proposition 2.6. All elements in $\pi_{8}^{S}$ are projective on $S^{m}$ for $m \equiv 3$ $(\bmod 4)$.

\section{Some projective properties of the $\operatorname{Im} J$} generators. Let $\beta$ be the generator of $\operatorname{Im} J$ in the $s$-stem, $s \equiv 0,1,3$ or $7(\bmod 8)$, and let $\alpha: S^{s+1} \rightarrow B O$ represent the corresponding generator of $\pi_{s+1}(B O)$. If $\alpha$ is thought of as an $m$-plane bundle over $S^{s+1}$, then the Thom space $\left(S^{s+1}\right)^{\alpha}$ is obtained from $S^{m}$ by attaching an $(m+s+1)$-cell using $\beta$ [9].

More generally let $\mu$ be an $m$-plane bundle over $X$. The inclusion of the base point $* \hookrightarrow X$ gives rise to a Puppe sequence [11]

$$
S^{m} \stackrel{{ }^{i_{\mu}}}{\longrightarrow} X^{\mu} \stackrel{\jmath_{\mu}}{\longrightarrow} \tilde{X}^{\mu} \stackrel{\theta_{\mu}}{\longrightarrow} S^{m+1} \longrightarrow \Sigma X^{\mu} \longrightarrow \cdots
$$


We call $\tilde{X}^{\mu}$ the reduced Thom space and $\theta_{\mu}$ the attaching map of $\mu$. With respect to cofibrations, Thom spaces have the following simple properties.

Proposition 3.1. Let $A \stackrel{f}{\rightarrow} B \stackrel{g}{\rightarrow} X$ be a cofibration sequence. Let $\eta=g^{*}(\mu), \epsilon=f^{*}(\eta)$ be the pullbacks of $\mu$. Then $A^{\epsilon} \rightarrow B^{\eta} \stackrel{h}{\rightarrow} \tilde{X}^{\mu}$, $\tilde{A}^{\epsilon} \rightarrow \tilde{B}^{\eta} \rightarrow \tilde{X}^{\mu}$ are cofibration sequences.

Here $h$ denotes the composite $B^{\eta} \rightarrow X^{\mu} \stackrel{I_{\mu}}{\longrightarrow} \tilde{X}^{\mu}$, and all other maps are naturally induced.

COROllary 3.2. The cofibre of $B^{\eta} \rightarrow X^{\mu}$ is $\Sigma \tilde{A}^{\epsilon}$.

Proof. Because the cofibre of $\tilde{B}^{\eta} \rightarrow \tilde{X}^{\mu}$ is $\Sigma \tilde{A}^{\epsilon}$, by (3.1).

Note also that $\epsilon$ is trivial, so $\Sigma \tilde{A}^{\epsilon}$ is just a suspension of $A$.

Next suppose that $X$ is the (reduced) suspension of a compact space $Y$. Let $b$ denote a base point. Let $\mu$ be given by the characteristic map $\chi_{\mu}: Y \rightarrow O(m)$. We can use $\chi_{\mu}$ to trivialise $\mu$ over $X-b$. Since $X-b \approx(Y-b) \times(0,1)$, the one-point compactification of $\mu \mid X-b$ can be identified with $\Sigma^{m+1} Y$. But on the other hand, this one-point compactification is clearly $\tilde{X}^{\mu}$. Thus we have

Proposition 3.3. Suppose that $X=\Sigma Y$ is compact and that the $m$-plane bundle $\mu$ on $X$ is given by the characteristic map $\chi_{\mu}: Y \rightarrow O(m)$. Then there is a homeomorphism $\omega_{\mu}: \Sigma^{m+1} Y \approx$ $\tilde{X}^{\mu}$. Furthermore, the attaching map $\theta_{\mu}$ corresponds to the image of $\chi_{\mu}$ under the composite homomorphism

$$
[Y, O(m)] \rightarrow\left[Y, \Omega^{m} S^{m}\right] \approx\left[\Sigma^{m} Y, S^{m}\right] \stackrel{\Sigma}{\rightarrow}\left[\Sigma^{m+1} Y, S^{m+1}\right] \stackrel{\omega_{\mu}^{*}}{\approx}\left[\tilde{X}^{\mu}, S^{m+1}\right] .
$$

COROllary 3.4. Suppose $X=\Sigma Y$ so that the (isomorphism classes of) $m$-plane bundles $\mu$ over $X$ form a group identifiable with $[Y, O(m)]$. Then $\theta_{r \mu}=r \theta_{\mu}$ for any integer $r$.

This corollary can be represented by the commutative diagram below.

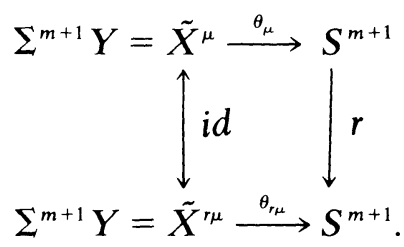


For more detailed discussion of reduced Thom spaces see [4] and [17, p. 230].

By studying Thom spaces over spheres and projective spaces, one can deduce certain projective properties of the generator $\beta \in$ $\operatorname{Im} J$. Specifically, consider the bundle $m \xi$ over $P^{s+1},(s \equiv 0,1,3$ or 7 $(\bmod 8))$, where $\xi=$ Hopf line bundle. We shall be interested in the case when $m \xi$ is not trivial over $P^{s+1}$, but is trivial over some skeleton $P^{t-1}$ with $t>\frac{1}{2}(s+1)$. We also need to suppose $t \not \equiv 0(\bmod 4)$. Under these circumstances $P_{t}^{s+1}$ is a suspension space $\Sigma Y, \widehat{K O}\left(P_{t}^{s+1}\right)$ is finite cyclic, and there is an $m$-plane bundle $\mu$ over $P_{t}^{s+1}$ which induces $m \xi$, under the collapsing map. Given $m$ and $s$, the existence of a skeleton $P^{t-1}$ satisfying all the above requirements can be guaranteed by the numerical condition $\phi\left[\frac{1}{2}(s+1)\right] \leqq \nu_{2}(m) \leqq \phi(s)$, where $\nu_{2}$ and $\phi$ are as explained in the introduction (see [1, Thm. 7.4]).

THEOREM 3.5. The generator $\beta$ of $\operatorname{Im} J$ in the $s$-stem is projective on $S^{m}$ whenever $\phi\left[\frac{1}{2}(s+1)\right] \leqq \nu_{2}(m) \leqq \phi(s)$.

Proof. First recall [2, Prop. (4.3)] that the Thom space of $m \xi$ over $P^{s+1}$ is $P_{m}^{m+s+1}$. Using the cofibration sequence

$$
\begin{aligned}
\left(P^{t-1}\right)^{m \xi} & \rightarrow\left(P^{s+1}\right)^{m \xi} \stackrel{h}{\rightarrow}\left(\widetilde{P_{t}^{s+1}}\right)^{\mu} \\
\mathbb{I N} & P_{m}^{m+t-1} \hookrightarrow \mathrm{P}_{m}^{m+s+1}
\end{aligned}
$$

of Proposition (3.1), we see that $\left(\widetilde{P_{t}^{s+1}}\right)^{\mu}$ is the truncated projective space $P_{m+t}^{m+s+1}$, and that $h$ is just a collapsing map between truncated projective spaces. Furthermore, since $h$ factors as $\left(P^{s+1}\right)^{m \xi} \rightarrow\left(P_{t}^{s+1}\right)^{\mu} \stackrel{j_{\mu}}{\rightarrow}\left(\widetilde{P_{t}^{s+1}}\right)^{\mu}$, and since $\theta_{\mu} j_{\mu} \simeq *($ see $(\#))$, we conclude that the composition $\theta_{\mu} c$ in

$$
\left(P^{s+1}\right)^{m \xi}=P_{m}^{m+s+1} \stackrel{c}{\rightarrow} P_{m+t}^{m+s+1}=\left(\tilde{P}_{t}^{s+1}\right)^{\mu} \stackrel{\theta_{\mu}}{\rightarrow} S^{m+1}
$$

is trivial.

Choose the integer $r$ so that the $m$-plane bundle $r \mu$ represents an element of order 2 in $\widetilde{K O}\left(P_{t}^{s+1}\right)$. As is well-known, $r \mu$ is then the pullback $c^{*}(\alpha)$ of an $m$-plane bundle $\alpha$ on $S^{s+1}$ representing a generator of $\widetilde{K O}\left(S^{s+1}\right)$. Recalling Corollary (3.4), we can now write down the following commutative diagram.

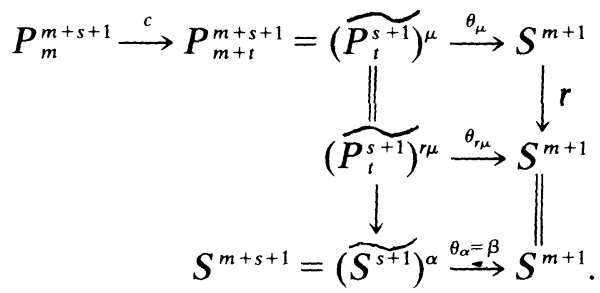


The space $P_{m}^{m+s+1}$ maps into each of the three spaces on the left column by a collapsing map $c$. Since $\theta_{\mu} c$ is trivial, we deduce by commutativity that $\theta_{r \mu} c$ is trivial, and hence the composition $P_{m}^{m+s+1} \stackrel{c}{\rightarrow} S^{m+s+1} \stackrel{\beta}{\rightarrow} S^{m+1}$ is trivial. Hence $\beta$ coextends in the cofibration sequence

$$
P_{m}^{m+s+1} \stackrel{c}{\rightarrow} S^{m+s+1} \stackrel{\Sigma \pi}{\rightarrow} \Sigma P_{m}^{m+s}
$$

i.e. we have a commutative diagram

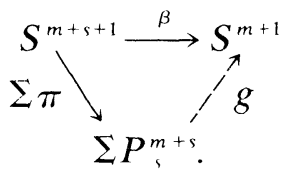

Desuspending this diagram, we see that $\beta$ is carried by $S^{m}$ projectively, as was to be proved.

REMARK. This theorem is not final. Rather it is a preparation for the much more comprehensive Theorem (7.1) below.

\section{Compression theorems for truncated projective} spaces. Let $P_{t}^{l}$ be a truncated projective space in which $t$ is assumed odd and larger than $l / 2$, so that $P_{t}^{l}$ is a suspension space $\Sigma Y$. If $l$ happens to be even, then (the class of) the identity map $1_{\Sigma Y}$ has finite order in the track group $[\Sigma Y, \Sigma Y]$. This order is a power of 2 , and has been determined by $\mathrm{H}$. Toda [16]. Furthermore, a map that equals a smaller power of 2 times $1_{\Sigma Y}$ can always be homotoped to a new map which "compresses" $\Sigma Y$ to a lower skeleton. In this section we present several theorems in this direction. These theorems lead to an immediate proof of some standard facts about generators of $\pi_{*}(O)$ and $\operatorname{Im} J$. See (4.3), (4.8) and (4.9).

THEOREM 4.1. Let $t$ be odd and $t>l / 2$ so that $P_{t}^{l}$ is a suspension space $\Sigma Y$. Then there exists a map

$$
\phi:\left(P_{t}^{l}, P_{t}^{l-1}\right) \rightarrow\left(P_{t}^{l-1}, P_{t}^{l-2}\right)
$$

such that, on passing to quotients, $\phi$ induces $\eta: S^{l} \rightarrow S^{l-1}$.

Proof. In case $l$ is even, the fundamental lemma of Toda [16, Lemma 2.1] says precisely that $2 \cdot 1_{\Sigma Y}$ can be homotoped to a map $\phi$ with the desired properties. 
In case $l$ is odd, $2 \cdot 1_{\Sigma Y}$ is of degree two and hence cannot be "compressed into a lower skeleton". However, it is not difficult to see that the composition

$$
P_{t}^{l} \stackrel{2 \cdot 1_{\Sigma Y}}{\longrightarrow} P_{t}^{l} \stackrel{i}{\longrightarrow} P_{t}^{l+1}
$$

can be homotoped to a map $\phi: P_{t}^{l} \rightarrow P_{t}^{l-1}$. (Consider the first-stage modified Postnikov resolution of the inclusion map $\left.P_{t}^{l-1} \rightarrow P_{t}^{l+1}\right)$ ! For any such $\phi$, the composite map $P_{t}^{l-1} \stackrel{1}{\rightarrow} P_{t}^{l} \stackrel{\phi}{\rightarrow} P_{t}^{l-1} \stackrel{c}{\rightarrow} S^{l-1}$ is necessarily trivial by virtue of cohomological degree. Hence $\phi$ can be chosen to satisfy $\phi\left(P_{t}^{l-1}\right) \subset P_{t}^{l-2}$.

To show that the quotient map $\phi: S^{l} \rightarrow S^{l-1}$ is nontrivial, take $\phi$ cellular, and induce a map $\phi^{\prime}: P_{l-2}^{l} \rightarrow P_{l-2}^{l-1}$ by collapsing the $(l-3)$ skeleta to a point. Then we have a commutative diagram

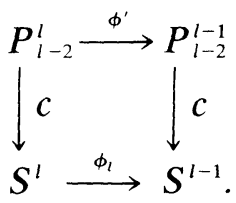

If $l \equiv 1$ or $5(\bmod 8)$, the nontriviality of $c \phi^{\prime}$ can be detected by the functor $\widehat{K O}$ or $\mathrm{KO}^{-4}$, so that $\phi_{l}=\eta$ in these cases. (Computation of the $K O$-groups offers no difficulty here. See [1].) As for the remaining cases $l \equiv 3,7(\bmod 8)$, it turns out that our present theorem holds even in a stronger form, which can be found in theorem (4.6) below. This concludes the proof.

COROLlary 4.2. For $t$ odd and $t>l / 2$, there is a commutative diagram

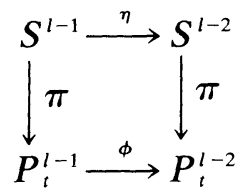

Proof. We have Puppe sequences $S^{l-1} \stackrel{\pi}{\rightarrow} P_{t}^{l-1} \stackrel{\iota}{\rightarrow} P_{t}^{l} \stackrel{c}{\rightarrow} S^{l}$ and $S^{l-2} \stackrel{\pi}{\rightarrow} P_{t}^{l-2} \stackrel{b}{\rightarrow} P_{t}^{l-1} \stackrel{c}{\rightarrow} S^{l-1}$ respectively. The map $\phi$ sends the middle terms of the first sequence into those of the second. The induced map $S^{l-1} \rightarrow S^{l-2}$ between the first terms must be $\eta$, by Theorem (4.1).

CoROllary 4.3. If $\rho$, denotes the generator of $\operatorname{Im} J$ in the $8 j-1$ stem, then the next two generators of $\operatorname{Im} J$ are $\rho_{,} \eta$ and $\rho_{j} \eta^{2}$, in the $8 j$ and $8 j+1$ stem respectively. 
Proof. It is easy to check that, when $l \equiv 1$ or $2(\bmod 8)$, the existence of $\phi$ in Theorem (4.1) implies that $\eta^{*}: \widetilde{K O}\left(S^{l-1}\right) \rightarrow \widetilde{K O}\left(S^{l}\right)$ sends generator to generator. But the generator of $K O\left(S^{l-1}\right)$ is just that of $\pi_{l-1}(B O)$ or $\pi_{l-2}(O)$. Thus our corollary is really an assertion about the homotopy generators of the infinite orthogonal group.

THEOREM 4.4. Assume $t$ is odd, $l \equiv 0,2,4(\bmod 8), l-8>t>$ $l / 2$. Then in the truncated projective space $P_{t}^{l}=\Sigma Y$, the map $16 \cdot 1_{\Sigma Y}$ can be homotoped to a map $\phi$ satisfying

$$
\phi\left(P_{t}^{l}, P_{t}^{l-2}\right) \subset\left(P_{t}^{l-8}, P_{t}^{l-9}\right) .
$$

Furthermore, for any such $\phi$, the quotient map $\phi_{\#}: P_{l-1}^{l} \rightarrow S^{l-8}$ is an extension of $8 \sigma: S^{i-1} \rightarrow S^{i-8}$.

This theorem can be represented diagrammatically by

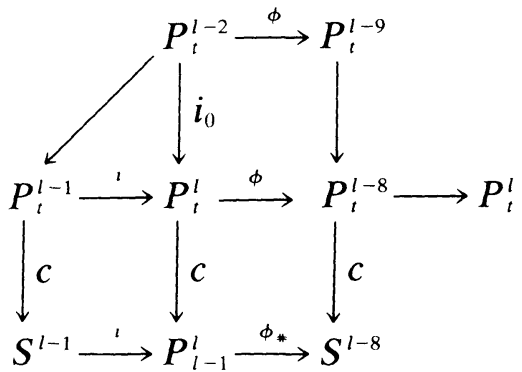

$$
\left(\phi_{\#} i=8 \sigma\right) \text {. }
$$

where the columns are cofibration sequences.

Proof. Make $16 \cdot 1_{\Sigma Y}: P_{t}^{l} \rightarrow P_{t}^{l}$ cellular and collapse to a point the $(l-8)$-skeleton in its domain and range. The resulting map is 16 times the identity map of $P_{l-7}^{l}$, which is trivial by Toda [16, Thm. 2.7]. From the cofibration sequence $P_{t}^{l-8} \stackrel{l}{\rightarrow} P_{t}^{l} \stackrel{c}{\rightarrow} P_{l-7}^{l}$, we see that $16 \cdot 1_{\Sigma Y}$ can be homotoped to a "compression map" $\phi: P_{t}^{l} \rightarrow P_{t}^{l-8}$. The following lemma guarantees that $\phi$ can be chosen to satisfy $\phi\left(P_{t}^{t-2}\right) \subset P_{t}^{t-9}$ as well.

Lemma 4.5. The composite $P_{t}^{l-2} \stackrel{t_{0}}{\rightarrow} P_{t}^{l} \stackrel{\phi}{\rightarrow} P_{t}^{l-8} \stackrel{c}{\rightarrow} S^{l-8}$ is homotopically trivial.

Assuming this lemma for the moment, we can now form the commutative diagram (III). If $\phi_{\#} i$ were trivial in that diagram, so would be $c \phi i: P_{t}^{l-1} \rightarrow S^{i-8}$, which allows $\phi$ to induce a map $\phi_{l}$, as shown below. 


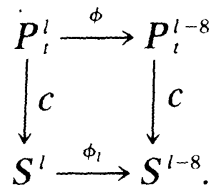

In $\tilde{K}$ or $\widetilde{K O}$ theory, $\phi^{*}$ is mono with cokernel $Z_{16}$, whereas $c^{*}$ has image $Z_{2}$. This would force $\phi_{l}^{*}: \tilde{K}\left(S^{l-8}\right) \rightarrow \tilde{K}\left(S^{l}\right)$ to be a nontrivial map $Z \rightarrow Z$ which is impossible because the finite group $\pi_{8}^{s}$ cannot operate nontrivially (by composition) on the torsion free groups $\pi_{*}(B U)$. On the other hand, $\phi_{*} i \in \pi_{l-1}\left(S^{l-8}\right)$ has order dividing 2 , because it extends to $P_{l-1}^{l}=S^{l-1} \cup_{2} e^{l}$. Therefore $\phi_{*} i$ equals $8 \sigma$, the only element of order 2 in the stable 7-stem. This completes the proof.

We now return to prove Lemma (4.5).

Case (i). $\quad l \equiv 0(\bmod 8)$.

Let $i_{1}$ and $i_{2}$ denote the inclusions $P_{t}^{l-2} \rightarrow P_{t}^{l-1}$ and $P_{t-2}^{l-8} \rightarrow P_{t}^{l-2}$ respectively. Also, denote sixteen times the identity map of $P_{t}^{l-2}$ simply by 16 . In spite of the fact that this is the restriction of $16 \cdot 1_{\Sigma Y}$, it is not necessarily true that $16 \simeq i_{2} \phi i_{0}$ as self-maps of $P_{t}^{l-2}$. However, by taking all maps and homotopies cellular, as one can clearly do, one certainly has $i_{1} \cdot 16 \simeq i_{1} i_{2} \phi i_{0}$. With this in mind, examine the following commutative diagram:

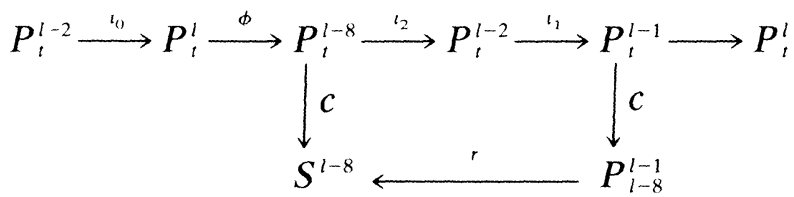

Here, the space $P_{l-8}^{l-1}$ is "co-reducible" in the sense that $P_{l-8}^{l-1} \simeq S^{l-8} \vee P_{l-7}^{l-1}$ [2, Thm. 6.2], and $r$ is a retraction map. Just like the compression of $16 \cdot 1_{\Sigma Y}$ into $\phi$, the map 16 of $P_{t}^{l-2}$ admits a compression into $P_{t}^{l-10}$, so that for dimensional reason any map from $P_{t}^{l-2}$ to $S^{l-8}$ factoring homotopically through 16 must be trivial. In particular, $c \phi i_{0}=r c i_{1} i_{2} \phi i_{0} \simeq\left(r c i_{1}\right) \cdot 16$ is trivial.

Case (ii). $\quad l \equiv 2(\bmod 8)$.

The $S$-dual of the map $c \phi i_{0}$ can be taken as a map $h: S^{N-l+8} \rightarrow P_{N-l+2}^{N-1}$, where $N+1=$ a high power of 2 [2, Thm. 3.3]. But $\pi_{N-1+8}\left(P_{N-l+2}^{N-t}\right)$ is the same as $\pi_{N-l+8}$ of the Stiefel manifold $V_{N-t+1, l-t-1}$, which is zero by the computations of [5]. Hence $c \phi i_{0}$ is trivial in this case.

Case (iii). $l \equiv 4(\bmod 8)$.

In this case we factor $i_{0}$ into a composition $P_{t}^{l-2} \stackrel{i^{\prime}}{\rightarrow} P_{t}^{l-1} \stackrel{t^{\prime \prime}}{\rightarrow} P_{t}^{l}$. The $S$-dual of $c \phi i_{0}$ can then be written as the following composite 


$$
S^{N-l+8} \stackrel{D c}{\longrightarrow} P_{N-l+8}^{N-t} \stackrel{D \phi}{\longrightarrow} P_{N-l}^{N-t} \stackrel{D i^{\prime \prime}}{\longrightarrow} P_{N-l+1}^{N-t} \stackrel{D^{\prime}}{\longrightarrow} P_{N-l+2}^{N-t}
$$

Applying $\pi_{N-1+8}$ and again using [5] as in Case (ii), we get homomorphisms

$$
Z \rightarrow Z_{2} \rightarrow Z_{8} \rightarrow Z_{16} \oplus Z_{4} \rightarrow Z_{8}
$$

It follows from algebraic considerations that the above composite must be trivial. Hence $c \phi i_{0} \simeq 0$.

By studying $2 \cdot 1_{\Sigma Y}$ instead of $16 \cdot 1_{\Sigma Y}$ in $\Sigma Y=P_{t}^{\prime}$, one can obtain the following analogous result. Compare [16, Lemma 2.2].

THEOREM 4.6. Assume $t$ is odd, $l \equiv 0(\bmod 4)$ and $l-2>t>$ l/2. Then in the truncated projective space $P_{t}^{l}=\Sigma Y$, the map $2 \cdot 1_{\Sigma Y}$ can be homotoped to a map $\phi$ satisfying

$$
\phi\left(P_{t}^{l}, P_{t}^{l-2}\right) \subset\left(P_{t}^{l-2}, P_{t}^{l-3}\right)
$$

Furthermore, for any such $\phi$, the quotient map $\phi_{\#}: P_{l-1}^{l} \rightarrow S^{l-2}$ is an extension of $\eta: S^{l-1} \rightarrow S^{l-2}$.

Coupling this theorem with (4.1), and using $4 \cdot 1_{\Sigma Y}=\left(2 \cdot 1_{\Sigma Y}\right)\left(2 \cdot 1_{\Sigma Y}\right)$, $8 \cdot 1_{\Sigma Y}=\left(2 \cdot 1_{\Sigma Y}\right)\left(4 \cdot 1_{\Sigma Y}\right)$, it is easy to deduce

Corollary 4.7. Let $P_{t}^{l}=\Sigma Y$ be as in Theorem (4.6). Then

(i) $4 \cdot 1_{\Sigma Y}$ can be homotoped to a map $\phi$ satisfying $\phi\left(P_{t}^{l}, P_{t}^{l-2}\right) \subset$ $\left(P_{t}^{l-3}, P_{t}^{l-4}\right)$. The quotient map $\phi_{\#}: P_{l-1}^{l} \rightarrow S^{l-3}$ of any such $\phi$ is an extension of $\eta^{2}: S^{l-1} \rightarrow S^{l-3}$;

(ii) $8 \cdot 1_{\Sigma_{Y}}$ can be homotoped to a map $\phi$ satisfying $\phi\left(P_{t}^{l}, P_{t}^{l-2}\right) \subset$ $\left(P_{t}^{l-4}, P_{t}^{l-5}\right)$. The quotient map $\phi_{\#}: P_{l-1}^{l} \rightarrow S^{l-4}$ of any such $\phi$ is an extension of $\eta^{3}=4 \nu: S^{l-1} \rightarrow S^{l-4}$.

As application of the above compression theorems we prove

Proposition 4.8. If $\rho$, denotes the generator of $\mathrm{Im} J$ in the $8 j-1$ stem, then $\rho_{l+1} \in\left\langle\rho_{l}, 8 \sigma, 2\right\rangle$.

Proof. Let $\bar{\rho}_{J}$ generate $\pi_{8_{j}}(B O)$. Set $l=8 j+8$ and choose $t$ odd in $(l / 2, l-8)$. Expand the two bottom squares of (III) to the following commutative diagram 


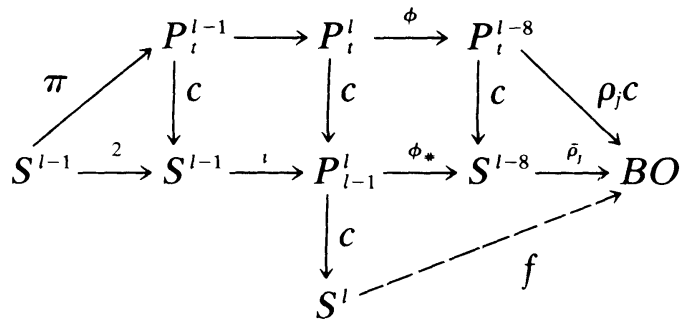

Notice that the mapping cone for 2 and $\pi$ are precisely $P_{l-1}^{l}$ and $P_{t}^{l}$, and that $f$ exists because any map from $S^{l-1}=S^{8_{1+7}}$ to $B O$ must be trivial. Indeed $f$ is none other than a representative of the Toda bracket $\left\langle\bar{\rho}_{j}, 8 \sigma, 2\right\rangle \subset \pi_{8++8}(B O)=Z$. Remembering that $\phi^{*}$ in $K O$-theory is a monomorphism with cokernel $Z_{16}$, it is routine to verify via commutativity that $f$ must represent an odd element. Taking indeterminacy into account, this establishes $\bar{\rho}_{\jmath+1} \in\left\langle\bar{\rho}_{\jmath}, 8 \sigma, 2\right\rangle$ and $\rho_{\jmath+1} \in\left\langle\rho_{l}, 8 \sigma, 2\right\rangle$ simultaneously.

Similarly, one can use Theorem (4.6) to prove

Proposition 4.9. After $\rho_{J} \eta^{2}$, the next generator $\xi_{\text {J }}$ of $\operatorname{Im} J$ in the $8 j+3$ stem lies in the Toda bracket $\left\langle\rho_{j} \eta^{2}, \eta, 2\right\rangle$.

\section{Composition properties (i) - primary com-} position.

THEOREM 5.1. Let $x \in \pi_{q}(X)$ be projective on the $(m-1)$ connected space $X$, where $q \leqq 2 m-4$. Then $x \eta$ is projective on $X$.

Proof. Let $t$ be the largest odd number $\leqq m$. According to Corollary (4.2), there is a map $\phi$ to make the following diagram commutative.

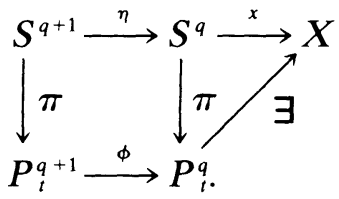

This establishes (5.1).

6. Composition properties (ii) - Toda brackets. We begin with some remarks on Toda brackets. In a commutative diagram such as 
(IV)

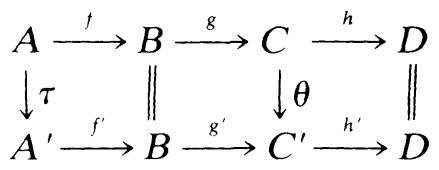

$$
\left(\begin{array}{c}
g f \simeq * \simeq h g \\
g^{\prime} f^{\prime} \simeq * \simeq h^{\prime} g^{\prime}
\end{array}\right)
$$

where two identity maps are involved, there need not be any naturality property relating $\langle h, g, f\rangle$ to $\left\langle h^{\prime}, g^{\prime}, f^{\prime}\right\rangle$, namely $\tau^{*}\left\langle h^{\prime}, g^{\prime}, f^{\prime}\right\rangle$ and $\langle h, g, f\rangle$ could be disjoint subsets in $[\Sigma A, D]$. Nevertheless, the situation can be salvaged by some additional hypothesis, such as assuming that the induced map $\tau_{+}$between the mapping cones $\mathscr{C}_{f}$ and $\mathscr{C}_{f^{\prime}}$ has certain nice properties.

Lemma 6.1. Suppose it is possible to expand (IV) to the following bigger commutative diagram

$(\mathrm{IV})^{\prime}$

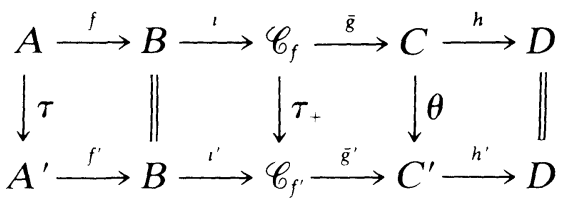

where $i, i^{\prime}$ are inclusions and $\bar{g}, \bar{g}^{\prime}$ are maps such that $\bar{g} i=g, \bar{g}^{\prime} i^{\prime}=$ $g^{\prime}$. Then there is a representative $\omega: \Sigma A^{\prime} \rightarrow D$ of $\left\langle h^{\prime}, g^{\prime}, f^{\prime}\right\rangle$ such that $\omega \cdot \Sigma \tau \in\langle h, g, f\rangle$.

The proof is routine and we omit it.

TheOREM 6.2. Let $x \in \pi_{q}(X)$ be projective on the $(m-1)$ connected space $X$, where $q \leqq 2 m-10$ and $q \equiv 3(\bmod 4)$. If the Toda bracket $\langle x, 8 \sigma, 2\rangle$ is defined, then all its representatives are projective on $X$.

Proof. Let $t$ be the largest odd number $\leqq m$ and let $h^{\prime}: P_{t}^{q} \rightarrow X$ be the map showing $x$ to be projective on $X$. In Theorem (4.4) we set $l=q+9$ and super-impose on top of diagram (III) a sub-diagram of the type (IV)', as shown below.

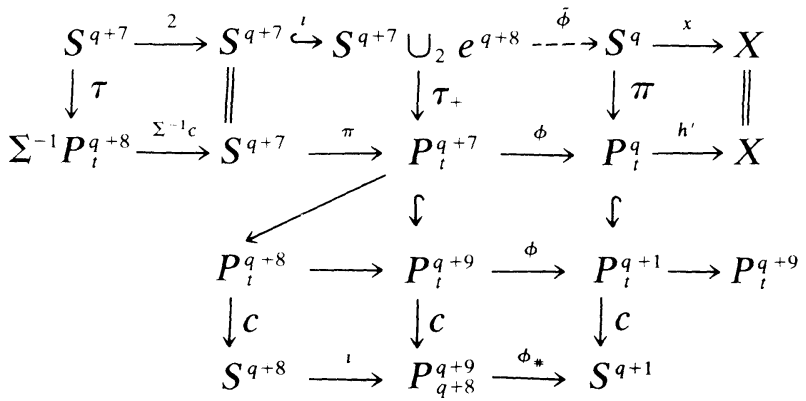


In this big diagram the square on the extreme left is the desuspension of

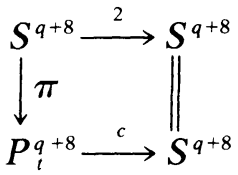

$(q+8$ being odd $)$

with $\Sigma^{-1} \pi$ simply abbreviated as $\tau$. The cofibre of $\Sigma^{-1} c$ is precisely $P_{t}^{q+7}$, as can be checked from the Puppe sequence [11] of $S^{q+7} \stackrel{\pi}{\longrightarrow} P_{t}^{q+7}$. Just as $P_{t}^{q+9}$ can be obtained from the subspace $P_{t}^{q+8}$ by attaching a cell via $\pi$, $P_{t}^{q+9}$ can also be obtained from the subspace $P_{t}^{q+7}$ by attaching two cells via $\tau_{+}$. Thus the vertical sequence starting with $S^{q+7} \cup_{2} e^{q+8}$ is a Puppe sequence, while the vertical sequence starting with $S^{q}$ is also one by construction. This induces the dotted arrow $\tilde{\phi}$, which can be thought of as a desuspension of $\phi_{\#}$.

One principal assertion of Theorem (4.4) is that $\phi_{*} i=8 \sigma$. By desuspension, $\tilde{\phi} i=8 \sigma$. Lemma (6.1) can now be applied to conclude that $\langle x, 8 \sigma, 2\rangle$ has a representative $f: S^{q+8} \rightarrow X$ factoring through $P_{t}^{q+8}$. Any other representative will differ from $f$ by sums of elements of the form $2 y$ or $x \theta$, where $y \in \pi_{q+8}(X), \theta \in \pi_{q+8}\left(S^{q}\right)$ are arbitrary. Since $q \equiv 3(\bmod 4), 2 y$ must be projective on $X$ by Proposition (2.1), and so must be $x \theta$, by Proposition (2.6). Consequently, all representatives of $\langle x, 8 \sigma, 2\rangle$ are projective on $X$, as was to be proved.

The following two theorems are proved in a similar fashion, using 4.6 and (4.7) (ii), respectively.

THEOREM 6.3. Let $x \in \pi_{q}(X)$ be projective on the $(m-1)$-connected space $X$, where $q \leqq 2 m-4$ and $q \equiv 1(\bmod 4)$. If the Toda bracket $\langle x, \eta, 2\rangle$ is defined, then all its representatives are projective on $X$.

THEOREM 6.4. Let $x \in \pi_{q}(X)$ be projective on the $(m-1)$-connected space $X$, where $q \leqq 2 m-6$ and $q \equiv 3(\bmod 4)$. If the Toda bracket $\langle x, 4 \nu, 2\rangle$ is defined, then all its representatives are projective on $X$.

7. Application to Whitehead products $\left[\iota_{n}, \beta\right], \beta \in$ Im $J$. In his memoir [7, §1] Mahowald studied the Whitehead product $\left[\iota_{n}, \theta\right]$ for $\theta \in \pi_{q}\left(S^{n}\right), q<2 n-2$, by using the fact that for all $k \geqq 1$,

$$
\left[\iota_{n}, \theta\right]=P_{k, n}\left(\Sigma^{n-1}\left(i_{*} \theta\right)\right) \in \pi_{q+n-1}\left(S^{n}\right),
$$

where $i: S^{n} \rightarrow P_{n}^{n+k-1}$ is the inclusion, $\Sigma^{n-1}$ is the suspension homomorphism from $\pi_{q}\left(P_{n}^{n+k-1}\right)$ to $\pi_{q+n-1}\left(\sum^{n-1} P_{n}^{n+k-1}\right)$, and $P_{k, n}$ is the "generalised Whitehead homomorphism" in the EHP sequence

$$
\pi_{q+n}\left(S^{n}\right) \stackrel{\Sigma^{k}}{\longrightarrow} \pi_{q+n+k}\left(S^{n+k}\right) \stackrel{I_{k, n}}{\longrightarrow} \pi_{q+n-1}\left(\sum^{n-1} P_{n}^{n+k-1}\right) \stackrel{P_{k, n}}{\longrightarrow} \pi_{q+n-1}\left(S^{n}\right)
$$


discussed by Toda in [15, Chapter XI]. Clearly, $i_{*}(\theta)=0$ implies $\left[\iota_{n}, \theta\right]=0$.

Thus to deduce vanishing theorems for $\left[\iota_{n}, \beta\right]$ where $\beta$ is a generator of $\operatorname{Im} J$, it suffices to show $\beta$ vanishes in $P_{n}$ (which implies $\beta$ vanishes in $P_{n}^{n+k-1}$ for $k$ sufficiently large). But this is equivalent, by Proposition (2.4), to $\beta$ being projective on a certain sphere. We already have a result of this nature in $\$ 3$. This result can now be significantly extended, using the composition theorems of $\$ 5$ and $\$ 6$.

Recall that $\beta$ can be one of the elements $\rho_{l}, \rho_{l} \eta, \rho_{l} \eta^{2}$ or $\xi_{\text {, }}$, in stem $8 j-1,8 j, 8 j+1$ or $8 j+3$ respectively. We now prove

THEOREM 7.1. The generator $\beta$ of $\operatorname{Im} J$ in the stable $s$-stem is projective on $S^{m}$, provided $3 \leqq \nu_{2}(m) \leqq \phi(s)$. (The stability condition $s \leqq m-2$ is implicitly assumed.)

This should be contrasted with Theorem (3.5), which requires the much stronger condition $\phi\left[\frac{1}{2}(s+1)\right] \leqq \nu_{2}(m) \leqq \phi(s)$.

Proof. We show that for any $m$ with $\nu_{2}(m) \geqq 3$, all $\operatorname{Im} J$ generators in the $s$-stem satisfying $\phi(s) \geqq \nu_{2}(m)$ are projective on $S^{m}$, as long as $s \leqq m-2$. First suppose that $\nu_{2}(m)$ has the form $4 j-$ $1(=\phi(8 j-1))$. By Theorem (3.5), $\rho_{\text {s }}$ is projective on $S^{m}$. By two applications of Theorem (5.1), $\rho_{\jmath} \eta$ and $\rho_{\jmath} \eta^{2}$ are projective on $S^{m}$. Since $\xi_{J} \in\left\langle\rho_{l} \eta^{2}, \eta, 2\right\rangle$ and $\rho_{l+1} \in\left\langle\rho_{l}, 8 \sigma, 2\right\rangle, \xi_{J}$ and $\rho_{l+1}$ must also be projective on $S^{m}$, by Theorems (6.3) and (6.2) respectively. Repeating the argument with $\rho_{J^{+1}}$, we deduce successively that all subsequent $\operatorname{Im} J$ generators are carried by $S^{m}$ projectively (as long as they are carried by it stably).

If $\nu_{2}(m) \neq 4 j-1$ we have to rely a bit more on Theorem (3.5). For example, if $3 \leqq \nu_{2}(m)=4 j-4(=\phi(8 j-8))$, then we can first use Theorem (3.5) to assert that $\rho_{\jmath-1} \eta, \rho_{\jmath-1} \eta^{2}, \xi_{j-1}$ and $\rho_{\jmath}$ are projective on $S^{m}$. The same is then true for all the subsequent generators, by the above argument. The cases $\nu_{2}(m)=4 j-3$ or $4 j-2$ are handled similarly.

The following table should help to illustrate Theorem (7.1).

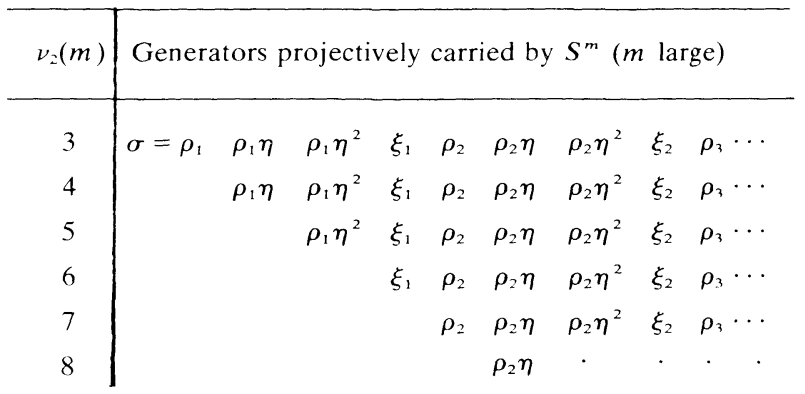


It is now a routine matter to translate (7.1) into Mahowald's theorem [7, p. 4, Theorem D].

THEOREM 7.2. The generator $\beta$ of $\operatorname{Im} J$ in the $s$-stem vanishes in $P_{n}$, and hence $\left[\iota_{n}, \beta\right]=0$ on $S^{n}$, provided $n$ and $s$ satisfy $3 \leqq \nu_{2}(n+s+2) \leqq$ $\phi(s)$.

REMARK. These vanishing results correspond to the cases marked by an " $x$ " in Mahowald's table of summary [7, p. 4]. They are the hardest cases to settle. The other vanishing results for $\left[\iota_{n}, \beta\right]$ in that table can also be established, rather effortlessly, by our approach.

REMARK (added in proof). In connection with Theorem (7.1), it can be shown without much difficulty that $\beta$ cannot be projective on $S^{m}$ if $\nu_{2}(m)>\phi(s)$.

\section{REFERENCES}

1. J. F. Adams, Vector fields on spheres, Ann. of Math., 75 (1962), 603-32.

2. M. F. Atiyah, Thom complexes, Proc. London Math. Soc., 11 (1961), 291-310.

3. G. E. Bredon, Equivariant stable stems, Bull. Amer. Math. Soc., 73 (1967), 269-73.

4. S. Feder and S. Gitler, Stable homotopy types of Thom complexes, Quart. J. Math. Oxford (2), 25 (1974), 143-49.

5. C. S. Hoo and M. E. Mahowald, Some homotopy groups of Stiefel manifolds, Bull. Amer. Math. Soc., 71 (1965), 661-67.

6. M. E. Mahowald, The order of the image of the $J$ homomorphism, Bull. Amer. Math. Soc., 76 (1970), 1310-13.

7. - The metastable homotopy of $S^{n}$, Memoir Amer. Math. Soc., No. 72 (1967).

8. R. J. Milgram, J. Strutt and P. Zvengrowski, Computing projective stable stems with the Adams spectral sequence, (to appear).

9. J. W. Milnor, On the Whitehead homomorphism, J. Bull. Amer. Math. Soc., 64 (1958), 79-82.

10. M. Mimura, J. Mukai, and G. Nishida, Representing elements of stable homotopy groups by symmetric maps, Osaka J. Math., 11 (1974), 105-111.

11. D. Puppe, Homotopiemengen und ihre induzierten Abbildungen I, Math. Z., 69 (1958), 299-344.

12. D. Quillen, The Adams conjecture, Topology, 10 (1971), 67-80.

13. E. Rees, Symmetric maps, J. London Math. Soc., 3 (1971), 267-72.

14. J. Strutt, Projective homotopy classes of spheres in the stable range, Bol. Soc. Mat. Mex., 16 (1971), 15-25.

15. H. Toda, Composition methods in homotopy groups of spheres, Ann. of Math. Studies, 49, Princeton, New Jersey.

16. - Order of the identity class of a suspension space, Ann. of Math., 78 (1963), 300-25.

17. T. C. T. Wall, Poincaré complexes: I, Ann. of Math., 86 (1967), 213-45.

Received October 1, 1975. Supported by the Multinational Program of Mathematics of the OAS.

Centro de Investigación y de Estudios Avanzados del Instituto Politécnico Nacional AND

UNIVERSITY OF British COLUMBIA

VANCOUVER, B. C., V6T 1W5, Canada 




\section{Pacific Journal of Mathematics}

Vol. 68, No. 1

March, 1977

Richard Julian Bagby, On $L^{p}, L^{q}$ multipliers of Fourier transforms . .......

Robert Beauwens and Jean-Jacques Van Binnebeek, Convergence theorems in

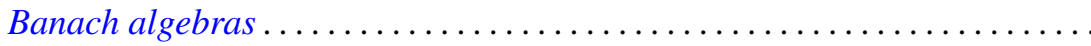

James Cyril Becker, Skew linear vector fields on spheres in the stable

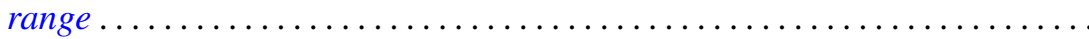

Michael James Beeson, Continuity and comprehension in intuitionistic formal

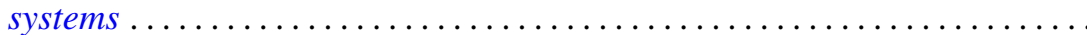

James K. Deveney, Generalized primitive elements for transcendental field

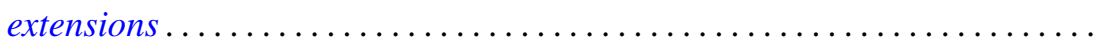

Samuel S. Feder, Samuel Carlos Gitler and K. Y. Lam, Composition properties

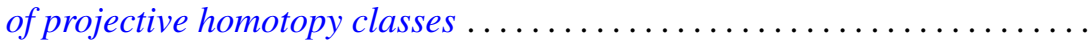

Nathan Jacob Fine, Tensor products of function rings under composition ......

Benno Fuchssteiner, Iterations and fixpoints . . . . . . . . . . . . . .

Wolfgang H. Heil, On punctured balls in manifolds

Shigeru Itoh, A random fixed point theorem for a multivalued contraction

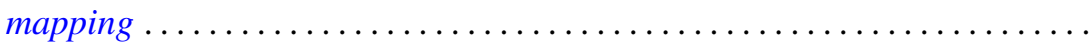

Nicolas P. Jewell, Continuity of module and higher derivations . . . . . . ......

Roger Dale Konyndyk, Residually central wreath products . . . . . . . . . . .

Linda M. Lesniak and John A. Roberts, On Ramsey theory and graphical

parameters.

Vo Thanh Liem, Some cellular subsets of the spheres.

Dieter Lutz, A perturbation theorem for spectral operators

P. H. Maserick, Moments of measures on convex bodies ... . . .

Stephen Joseph McAdam, Unmixed 2-dimensional local domains . .

D. B. McAlister and Norman R. Reilly, E-unitary covers for inverse semigroups...

William H. Meeks, III and Julie Patrusky, Representing codimension-one

homology classes by embedded submanifolds . . .

Premalata Mohapatro, Generalised quasi-Nörlund summability . .

Takahiko Nakazi, Superalgebras of weak-*Dirichlet algebras .

Catherine Louise Olsen, Norms of compact perturbations of operators .

William Henry Ruckle, Absolutely divergent series and isomorphism of

subspaces. II.

Bernard Russo, On the Hausdorff-Young theorem for integral operators .

Arthur Argyle Sagle and J. R. Schumi, Anti-commutative algebras and

homogeneous spaces with multiplications ............

Robert Evert Stong, Stiefel-Whitney classes of manifolds .

D. Suryanarayana, On a theorem of Apostol concerning Möbius functions of

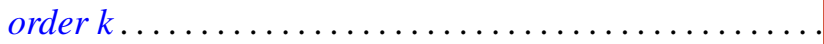

Yoshio Tanaka, On closedness of $C$ - and $C^{*}$-embeddings . . 